DOI 10.31489/2019No2/26-30

UDC 620.13 .40

\title{
EXPERIMENTAL STUDY OF CHANGES IN THE STRUCTURE OF POLYMER COMPOSITE MATERIAL BY COMPUTATIONAL X-RAY TOMOGRAPHY
}

\author{
Yurov V.M. ${ }^{1}$, Goncharenko V.I. ${ }^{2,3}$, Vasiliev S.L. ${ }^{2}$, Dmitriev S.A. ${ }^{2}$, Yurgenson S.A. ${ }^{2}$ \\ 1 E.A. Buketov Karaganda State University, Karaganda, Kazakhstan, exciton@list.ru \\ ${ }^{2}$ Moscow Aviation Institute (National Research University), Moscow, Russia, fvo@mai.ru \\ ${ }^{3}$ Institute of Control Sciences. V.A. Trapeznikova, RAS, Moscow, Russia, fvo@mai.ru
}

\begin{abstract}
In article on the basis of the method of computing x-ray tomography the technique of assessing changes in the state structure of polymeric composite material under the dead load. The results of the analysis of structure changes for carbon fiber samples at the setting stage of loading are presented. Offered exploratory procedure allows to fracture propagation under the force action and to analyze the material changes depending on the presence and load levels. In addition, quantitative parameters are introduced to compare the tendency of the material to accumulate damage in various technological processes. The results of the test research show that the proposed method allows for high accuracy to analyze the behavior of the material under load and identify typical trends for the type of material and laying.
\end{abstract}

Keywords: composite material, X-ray computer tomography, nondestructive testing, aviation structure.

\section{Introduction}

At this moment in time production engineering of elements and item of equipment of composite material (CM) are developing ahead of schedule in almost all industrialized countries. Feature of CM is that they are not monolithic material, such as metals. KM is a structure which created in the process of manufacturing a product [1-3]. There is a problem of an objective assessment of quality of KM, possibility of application of various physical methods for control of their quality. Computational and analytical methods of research do not always allow obtain a complete picture of all the effects arising during servicing in polymer composite materials (PCM) $[4,5]$. Conformance assessment of materials, blank part, and final production can be carried out using a variety of laboratory and industrial equipment. One of the methods of flaw detection and analysis of size dimensions of the product is the method of computational X-ray tomography [5].

Manufacturing computational X-ray tomography (CXT) is a highly effective method of nondestructive radiation control, combining the capabilities of $\mathrm{X}$-ray radiation and digital technology, which allows obtain flat sections and three-dimensional images of controlled products. On-hand status of such equipment at the "Production planning and quality control" department of the Moscow aviation Institute (national research University) allows to carry out some work on the conformity assessment of military products $[6,7]$.

The proposed work is at the junction of several areas - the development of methods of application of high-energy tomography in the mechanics of structurally inhomogeneous materials and technological design [8-16]. Within these areas are suggested to develop perspective methods of research of new materials, correct methods of designing products from polymeric composite materials and to develop methods for computational and analytical evaluation of the product behavior during operation based on process defects.

The paper [7] describes the method of using CXT in solving problems of research the state of the structure of the PCM under load. The main data obtained in this work are related to the assessment of crack development in the material and measurements based on computer modeling of 
its contribution to the reduction of the sample strength. At the same time, the research method proposed in this paper allows not only to assess the crack development under force action, but also to analyze the material changes depending on the presence and level of loading. Quantitative parameters are introduced to compare the tendency of the material to accumulate damage in various technological processes.

\section{Experimental procedure}

For a measurement of change in the power action stand, allowing to apply to the samples uniaxial force action directly on the desktop of the X-ray tomography, had been engineered and made. At the figure 1 we can see diagram of the research using this stand. The initial fixed parameters are the characteristics of the sample and its mechanical properties, which are used to select the loading steps (drawing up the plan of the experiment).

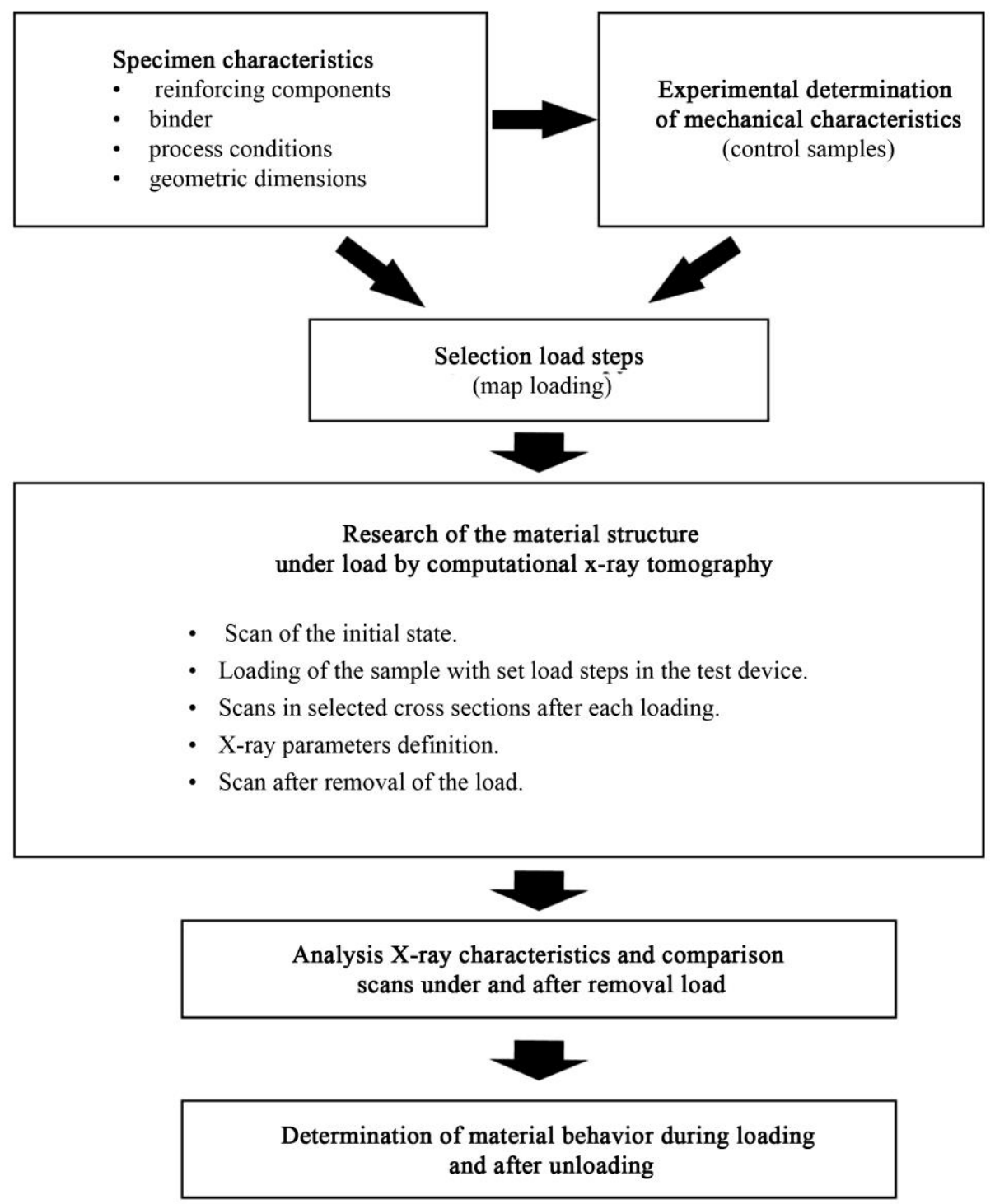

Fig.1. Study design

Test operation is determining the basic state of the material-the tomographic parameters that are considered to be the starting point for a this sample (selected technology, installation and types of binder and filler). And then step-by-step loading is performed for the selected load levels. At 
every step the state of the material is fixed and tomographic parameters are determined, which are compared with the base state, which allows to evaluate the changes occurring in the material. Growth of density anomalies associated with both loosening of the material and its local compaction. The maximum value of the linear attenuation coefficient (LAC) is monitored to track the compaction of individual sections.

At every step of increasing the load, the following actions are performed:

- Each test sample is installed in a stand for force interaction on the sample. Initial strain measurement and preliminary scanning of all selected sections are carried out;

- A load is applied to the sample, the value of which corresponds to the first step of loading, deformations in the sample are measured and scanning is carried out;

- The sample is unloaded (complete removal of extension force) and immediately carried out deformation measurement and scanning;

- After a lapse of time after removal of load carried out repeating deformation measurement;

- Unloading and further loading of the sample is carried out in accordance with the plan of the experiment;

- Then the sample is loaded to the level of the second step and the test procedure is repeated.

- Number of steps of increasing the load are repeating while sample do not be destroyed (or reaching the limit to fit the force) or before deformations appear in the sample, indicating a complete loss of load-bearing capacity (expected for samples with oblique-angle reinforcement scheme) [10].

As tomographic parameters the criteria of correlation between the state of the structure of the sample material and the reconstructed scanning results are accepted:

1. The average value attenuation coefficient be a descriptor of structural density in the i-th scanned section after the $\mathrm{j}$-th loading ( $\mathrm{j}=0$ - before loading) and is directly proportional to the increase in the number of secondary damage per unit area of the section;

2. Maximum and minimum value of LAC, which characterize growth process of individual defects and local compaction in the material structure;

3. Root-mean-square deviation (RMS) of the value of LAC in the scanned section characterizes the amplitude of the absolute deviation of the LCO from the most probable value, the increase in the absolute value of the LCO confirms the increase in the number of secondary damages per unit area.

\section{Results of the experiment and their discussion}

As test pattern applied specimen series UP-3 (with typical laying $0 / \pm 45 / 90$ ) of carbon tape UOL-300-1 (TU 1916-167-05763346-96 production LLC "Argon") and the binder EPS-I-108 (TU 2225-047-17411121-2012 production LLC "Superplast"), obtained by impregnation and molding under a double vacuum bag. Research was being conducted in the 40 sections of the operating space with step for 5 millimeters for debugging parameters of scanning and analysis of the frequency of occurrence of values. In appropriate cases thickener or defect of anyone type can be input into researching space of specimen.

The research of the sample was carried out at four levels of tensile load $-5 \%, 20 \%, 30 \%, 40$ $\%$ from the destructive load, as well as its further full unloading. As a result, the graphs of the deviation of the controlled parameters from the initial state of the sample were obtained, presented in figure 2. Analysis of the change in the cross-sectional area of the sample was not carried out because of its minor fluctuations - sectional area was not more than $0.5 \%$, which may be caused by the heterogeneity of the sample surface. Based on the results for the selected installation and types of materials, the following conclusions are made:

- When applying up to $30 \%$ of the breaking load the sample structure changes with a small step and does not undergo significant changes. There is an ordering of the structure of the material, 
as evidenced by a decrease in the deviation of the maximum values of the LAC and slight fluctuations in the deviation of the minimum value, which shows a variable growth of the primary defect. Growth deviations RMS in this case is associated with the most fluctuations in minimum values of LAC compared to the maximum, which indicates the early growth of the primary defect that causes the seal adjacent layers (decreases deviation of the average value of LAC);
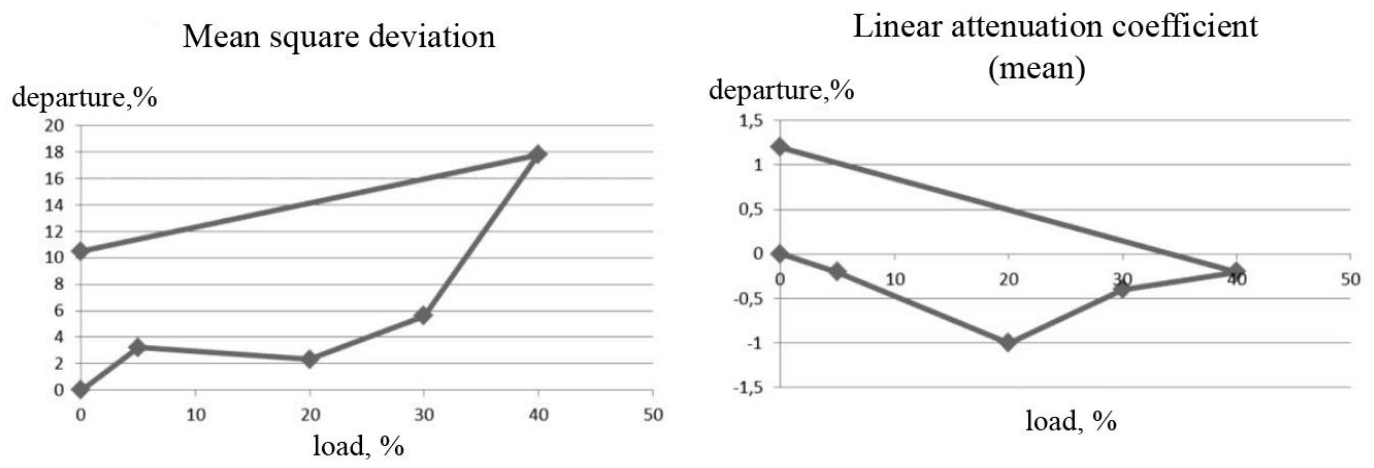

Linear attenuation coefficient

Linear attenuation coefficient (minimum)
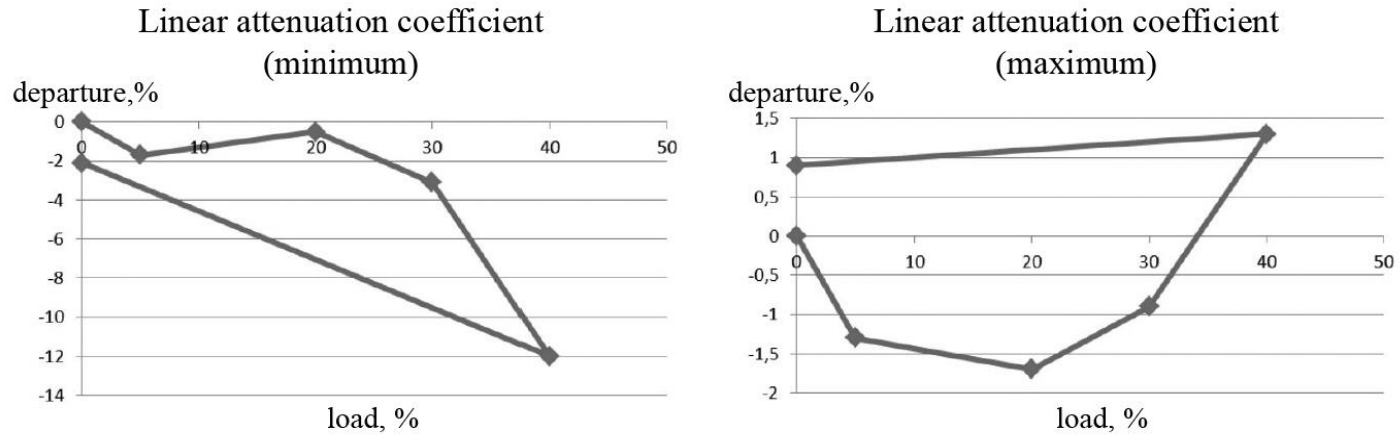

Fig. 2. Deviation of controlled parameters from the initial state depending on the applied load.

- When applying up to $40 \%$ of the breaking load is reached, a significant change in the state of the structure of the material is observed. The increase in the deviation of the RMS value from the initial value when $40 \%$ of the destructive load is reached is 2 times higher than the same indicator at $30 \%$ of the destructive load. This is caused by a significant increase in the primary defect (the minimum value of LAC increases by 3 times compared to $30 \%$ of the breaking load), which leads to compaction of the surrounding material. This conclusion is confirmed by the behavior of the average value of Loco, which is $40 \%$ of the destructive load almost corresponds to the initial state of the sample, while the growth of RMS. After unloading of the sample, residual variation are observed, which are characterized by a local compaction of the sample structure, significant in comparison with the loaded state. The General behavior of the material structure after unloading is characterized by an increase in density variations in the sample (the deviation of the RMS from the initial state is $10 \%$ ) and a slight compaction of the material structure (the average value of the LAC increased by $1 \%$ ), which may mean a decrease in the number of primary defects in the sample. According to the results of the test research on this sample, the following conclusions can be made:

- for this placing and material characteristic considerable increase mainstream defect after reaching a certain load (in this case it was $30 \%$ of the destructive load);

- when the load is applied, the growth of the local primary defect occurs, while smaller defects are closed, and the structure of the material becomes less loose;

- after unloading the sample, there is a general compaction of the material structure, but at the same time there is a disclosure of the mainstream defect [12], which in the case of flaw detection after removing the load has a much smaller geometric dimensions compared to the loaded state. 


\section{Conclusion}

The suggested procedure shows that the proposed technique helps high accuracy carry out an analysis of the material response when load is applied, and identify trends specific to the type of material and styling in question. Comparison of the initial, loaded, and unloaded state of the sample indicates the need for such a study for different types of materials in order to identify the characteristic trends in the main cracks.

The increase mainstream defect and less the influence of local defects on the structure of the material was obtained for this placing and material characteristic. At the same time local defects on the structure of the material must be research same the mainstream defect. The volume of porosity and moisture saturation may be changed in real structure by comparison with elementary samples was researched in the certification tests. The local changes of structure material was had on the low load, that mean that real volume of porosity (therefore and moisture saturation) can be higher and mechanical characteristics low than design value. The possibility of numerical evaluation of the processes of changing the structure of the composite material allows us to clarify the existing results and methods of finite element calculation. The methods of damage summation processes in CM can be improve with the experimental data sufficiency.

\section{REFERENCES}

1 Mikhailin Yu.A. Structural polymer composite materials, 2nd edition. Moscow, 2008, 822 p.

2 Goncharenko V.I., Oleshko V.S. Calculations of Tool Hardness in the Aviation Industry. Russian Engineering Research. 2017, Vol. 37, No. 6, pp. 554-556. DOI: 10.3103/S1068798X17060119.

3 Goncharenko V.I., Oleshko V.S. Determining the surface energy of tools in the aviation industry. Russian Engineering Research. 2017 Vol. 37, No. 7, pp. 628-630. DOI: 10.3103/S1068798X17070127.

4 Lurie S.A., Dudchenko A. Introduction to the mechanics of nanocomposites. Moscow, 2010, $160 \mathrm{p}$.

5 Klyuev V.V., Sosin F.R., Kovalev A.V. Non-destructive testing and diagnostics. Weinberg and others. 2nd edition. Moscow, Engineering, 2003, 656 p.

6 Boicov B.V., Vasilyev S.L., Hromachev A.G., Jurgenson S.A. Non-destructive testing methods used for structures made of advanced composite materials. Labor of MAI: Electronic Journal. 27.12.2011. Issue 49.

7 Vasilyev S.L., Artemyev A.V., Jurgenson S.A. Analysis of the impact of static load on the structure of polymer composite material by computational x-ray tomography. Proceedings of the XIntern. Conference on nonequilibrium processes in nozzles and jets, Alushta, May 25-31 2014. Moscow, 2014, pp. 543-545.

8 Nikishkov Y., Airoldi L., Makeev A. Measurement of Voids in Composites by X-Ray Computed Tomography. Composites Science and Technology, 2013, 89, pp. 89-97.

9 Fuji T., Zako M. Fracture mechanics of composite materials. Moscow, Mir, 1982, 232p.

10 Brautman L., Krok R. Composite materials. Fracture and fatigue. Moscow, 1978. Vol. 5, 483 p.

11 Potrakhov N.N., Anoshkin A.N., Zuiko V.Yu. and others. Computational and experimental evaluation of the strength of the composite frame segment using the method of in-situ X-ray inspection. Bulletin of the Perm national research Polytechnic University. Mechanics. 2017, No. 1, pp.118 - 133.

12 Na W.J., Ahn H.C., Park K.M., Kang H.M., Yu W.R. In-situ damage monitoring of textile composites using x-ray computed tomography. Proceedings of the ECCM15: 15th European conference on composite materials. Italy, Venice, 2012, pp. 229 - 236.

13 Withers P.J., Preuss M. Fatigue and Damage in Structural Materials Studied by X-Ray Tomography. Annual Reviews. 2012, Vol. 42, p. 81-103. DOI: 10.1146/annurev-matsci-070511-15511.

14 Brault R., Germaneau A., Dupré C., Doumalin P., Mistou S., Fazzini M. In-situ Analysis of Laminated Composite Materials by X-ray Micro-Computed Tomography and Digital Volume Correlation. Experimental Mechanics. 2013, Vol. 53, No. 7, p. 1143-1151. DOI: 10.1007/s11340-013-9730-9.

15 Yurov V.M., Oleshko V.S. The impact of the environment on the contact potential difference of metal machine parts. Eurasian Physical Technical Journal. 2019. Vol. 16, No. 1 (31). pp. 99-108.

16 Oleshko V.S. Optimal Number of Duralumin Samples in Determining the Surface Energy. Russian Engineering Research. 2019, Vol. 39, No. 3, pp. 272-275. https://doi.org/10.3103/S1068798X19030183. 